Archived version from NCDOCKS Institutional Repository http://libres.uncg.edu/ir/asu/

\title{
Appalachïan
}

B O O N E, N O R T H C A R O L I N A

\section{Relationships Among Subjective and Objective Measures of Tongue Strength and Oral Phase Swallowing Impairments}

Authors:

Heather M. Clark, Pamela A. Henson, William D. Barber, Julie A. G. Stierwalt, Michael Sherrill

\begin{abstract}
A growing literature documents the relationship between tongue strength and oral phase swallowing function. Objective measures of strength have been recommended as more valid and reliable than subjective measures for the assessment of tongue function, yet subjective measures remain the more commonly used clinical method for assessing tongue strength. This study assessed the relationships among subjective and objective measures of tongue strength and oral phase swallowing impairments. Both subjective and objective measures of tongue strength were observed to be good predictors of the presence of oral phase swallowing impairments. The specific oral phase swallowing functions of bolus manipulation, mastication, and clearance were moderately correlated with subjective ratings of tongue strength. Experienced and inexperienced raters appeared to judge tongue strength differently, with the ratings of experienced raters being more predictive of swallowing function.
\end{abstract}

Clark, Heather, Henson, Pamela, Barber, William, Stierwalt, Julie. \& Sherrill, Michael. (2003). Relationships among subjective and objective measures of tongue strength and oral phase swallowing impairments, American Journal of Speech-Language Pathology [12(1),pp. 40 - 50.] Published by the American Speech-Language-Hearing Association (ISSN: 1058-0360). 
Clark, H. M., Henson, P.A., Barber, W. D., Stierwalt, J. A. S. \& Sherrill, M. (2003). Relationships among subjective and objective measures of tongue strength and oral phase swallowing impairments, American Journal of SpeechLanguage Pathology, (Feb 2003) 12(1), 40 - 50. Published by the American Speech-Language-Hearing Association (ISSN: 1058-0360).

\title{
Relationships Among Subjective and Objective Measures of Tongue Strength and Oral Phase Swallowing Impairments
}

\author{
Heather M. Clark, Pamela A. Henson, William D. Barber, Julie A. G. Stierwalt, Michael \\ Sherrill
}

\begin{abstract}
A growing literature documents the relationship between tongue strength and oral phase swallowing function. Objective measures of strength have been recommended as more valid and reliable than subjective measures for the assessment of tongue function, yet subjective measures remain the more commonly used clinical method for assessing tongue strength. This study assessed the relationships among subjective and objective measures of tongue strength and oral phase swallowing impairments. Both subjective and objective measures of tongue strength were observed to be good predictors of the presence of oral phase swallowing impairments. The specific oral phase swallowing functions of bolus manipulation, mastication, and clearance were moderately correlated with subjective ratings of tongue strength. Experienced and inexperienced raters appeared to judge tongue strength differently, with the ratings of experienced raters being more predictive of swallowing function.
\end{abstract}




\section{ARTICLE}

Intact lingual function is commonly understood to be necessary for normal swallowing function. The tongue plays a role in maintaining and manipulating a cohesive bolus, in masticating, and in propelling the bolus out of the oral cavity and through the pharynx (Logemann, 1998). Two studies have documented the relationship between reduced tongue strength and impaired swallowing function. Reddy, Costarella, Grotz, and Canilang (1990) found that participants with dysphagia exhibited reduced lateral tongue force when compared with control participants. Robinovitch, Herschler, and Romilly (1991) reported that a patient with dysphagia exhibited reduced left horizontal tongue force.

These studies leave several questions unanswered regarding the relationship between tongue strength and swallowing function. First, it is unclear which aspects of swallowing function were influenced by reduced tongue strength. Reddy et al. (1990) reported that the participants with dysphagia in their study were able to swallow liquids, but not solids. From this description, it is unclear whether the participants exhibited impairments in the oral and/or pharyngeal phases of swallow. The participant described by Robinovitch et al. (1991) exhibited oral phase dysphagia, but the precise nature of the impairment was not described. Thus, although it is intuitive that patients with reduced tongue strength might exhibit reduced bolus control, impaired mastication, and reduced oral and pharyngeal clearance, the precise nature of these relationships has not been documented in the literature.

A second limitation of the extant literature is the small group of patients with dysphagia studied: Robinovitch et al. (1991) reported results from 2 participants with dysphagia, and Reddy et al. (1990) reported on 12 participants. A related limitation is the small size of the control groups to which the patients with dysphagia were compared. Both Robinovitch et al. and Reddy et al. reported a small number of control participants (7 and 12, respectively). These are exceedingly small samples on which to base judgments of normal tongue strength, especially as neither of the above studies cited additional normative data for the tools used.

A final issue concerning the above reports is that both studies employed objective measures of tongue strength. Researchers have argued that objective measures are more accurate and reliable than subjective measures (e.g., having the patient push with his/her tongue against a tongue blade while the clinician provides resistance, Luschei, 1991; Reddy et al., 1990; Robinovitch et al., 1991; Sukthankar, Reddy, Canilang, Stephenson, \& Thomas, 1994). Clinical instruments for objective measurement of tongue strength are becoming more available (e.g., the Kay Swallowing Signals Lab and the lowa Oral Performance Instrument). However, most clinicians continue to rely on subjective 
measures of tongue strength, even though the relationship between such measures and oral phase swallowing function has yet to be studied.

The present study addressed the limitations identified above. We examined the relationships among subjective and objective measures of tongue strength and several different aspects of oral phase swallowing function [1] in 63 participants with dysphagia. The objective measures of swallowing function were obtained using the lowa Oral Performance Instrument (IOPI), a device for which normative data (Robin \& Luschei, 1992) and additional normal performance ranges have been reported (Crow \& Ship, 1996; Robbins, Levine, Wood, Roecker, \& Luschei, 1995; Robin, Goel, Somodi, \& Luschei, 1992; Robin, Somodi, \& Luschei, 1991; Solomon, Lorell, Robin, Rodnitzky, \& Luschei, 1995; Solomon, Robin, \& Luschei, 2000; Stierwalt, Robin, Solomon, Weiss, \& Max, 1996).

Our study also included subjective measures of tongue strength obtained during a standard oral-motor examination, as well as descriptions of several aspects of oral phase swallowing function. The subjective measures of tongue strength incorporated judgments during protrusion and lateral movements. We hypothesized that these measures would be associated with impairments in bolus control, mastication, and bolus propulsion. We further hypothesized that objective measures obtained with the IOPI, which assesses tongue strength during anterior tongue elevation, would be related to the presence of oral residue secondary to impaired bolus propulsion.

A secondary issue was also of interest in this study. Previous research using objective measures of tongue strength assumed that such measures were more valid and reliable than subjective measures. However, none of these studies directly examined the relationship between subjective and objective measures of tongue strength. Furthermore, despite widespread clinical use of subjective measures of tongue strength (e.g., Duffy, 1995; Logemann, 1998), no studies have investigated factors affecting the accuracy of strength ratings. Our study investigated the relationship between subjective ratings of tongue strength obtained during a standard oral-motor examination, and objective measures of tongue strength. Additionally, the impact of examiner experience on the relationship between subjective and objective measures of tongue strength was evaluated.

In summary, this study addressed the primary question: What are the relationships among subjective and objective measures of tongue strength and various aspects of oral phase swallowing function? Secondarily, we asked: How are these relationships affected by examiner experience? 


\section{Method}

This study was conducted using data collected prospectively during the standard operation of a clinical practice. Participant selection and assessment procedures were minimally controlled to maintain maximum ecological validity.

\section{Participants}

This research was conducted with the approval of the institutional review boards of both the clinical facility and the cooperating university. Participants were recruited from a group of patients referred to the speech-language pathology department of a small regional hospital for a swallowing evaluation. Both inpatients and outpatients were included in the study. All patients who were able to complete the tasks required for the study and who agreed to participate were included.

A total of 63 patients participated in the study. They ranged in age from 19 to 95 years, with a mean age of 70.25 years. The mean age of the 28 male participants was 72.5 years (range $=19-95$ ), and the mean age of the 35 female participants was 73 years (range $=37-91$ ). Participants ranged from 1 day to 3 years postonset of dysphagia, with a median of 14 days postonset. Table 1 lists the referral diagnoses for the participants. Because this study was concerned with the relationship between tongue strength and oral phase swallowing function, regardless of etiology, referral diagnosis was not included as an independent variable.

TABLE 1. Participant descriptions.

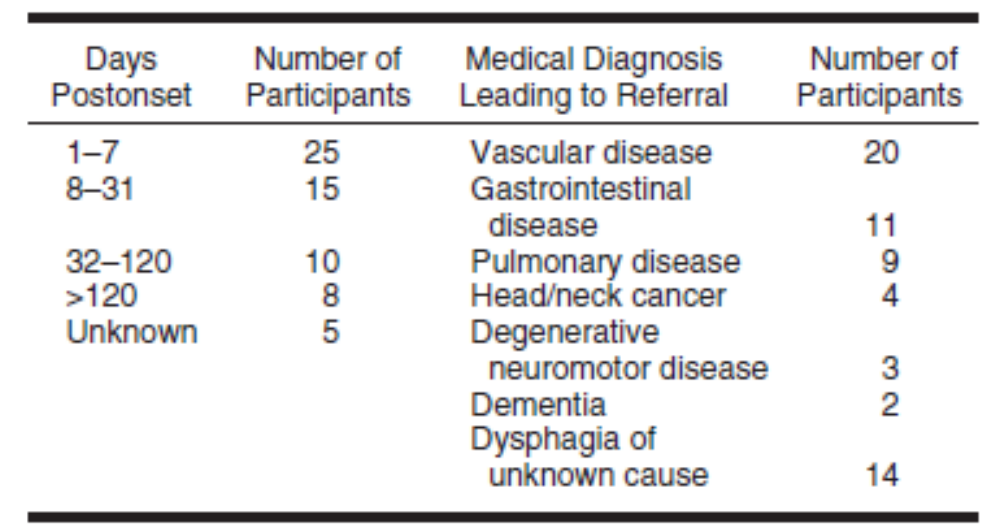




\section{Examiners}

The examiners for the study were divided into two groups: experienced and inexperienced. The experienced examiners included two certified speech-language pathologists (the first and second authors), with 8 and 14 years of experience in the assessment of swallowing function, respectively. The inexperienced examiners included nine graduate students completing their fourth semester of clinical practicum experience. The students had no prior experience subjectively judging tongue strength.

\section{Procedures}

Subjective Measures. All participants were evaluated using the standard clinical protocol adopted by the cooperating facility. Specifically, the swallowing examination included an assessment of oral structure and function, as well as observations of swallowing function. During the oral motor assessment, subjective measures of tongue strength were obtained by having the participant press with the tip of his/her tongue against a tongue blade, with resistance provided by the examiner. This method was used to assess the strength of both protrusion and lateralization. The examiners rated tongue strength as normal, slightly weak, moderately weak, or severely weak. Because standardized training in the subjective rating of tongue strength is not common for speech-language pathologists, specific training in rating tongue strength was omitted in this study in order to determine the reliability of subjective measures under typical clinical conditions. 


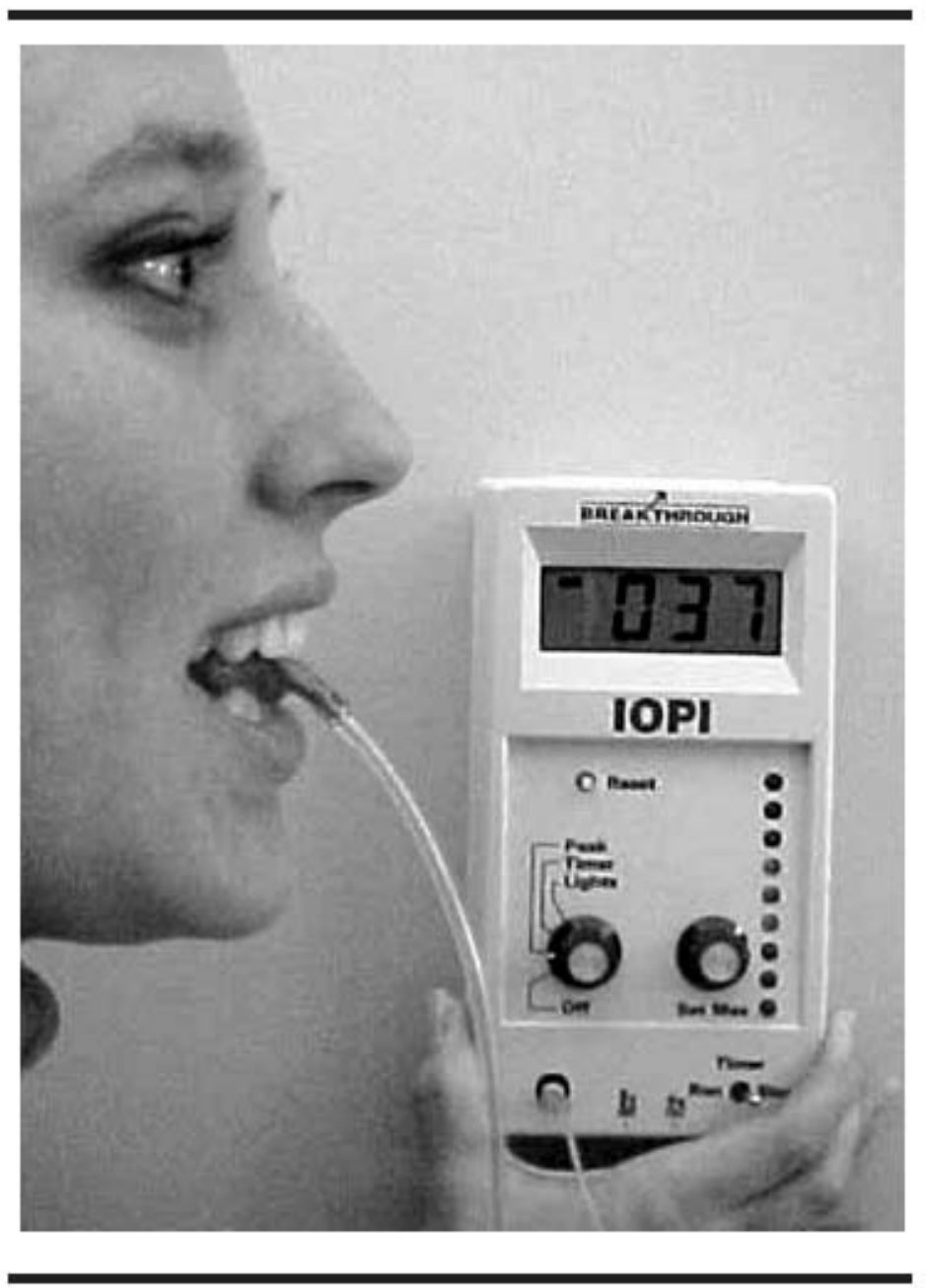

Objective Measures. In addition to the subjective measures of tongue strength, objective measures were obtained using the IOPI, which has been described in several previous works (Robbins et al., 1995; Robin et al., 1991, 1992). The IOPI consists of a battery-operated pressure transducer and amplifier that displays a digital reading in kilopascals $(\mathrm{kPa})$ reflecting pressures exerted on an air-filled bulb (Figure 1). To measure tongue strength, the bulb was positioned behind the participant's incisors so that the middle portion of the bulb was in contact with the alveolar ridge. Each participant was instructed to "push as hard as possible" with his/her tongue against the bulb. Three motivated trials were conducted and recorded. Only valid trials (i.e., the bulb was properly positioned and pressure was applied only by the tongue) were recorded.

Tongue strength obtained using the IOPI has traditionally been defined as the highest pressure generated across three motivated trials (Robbins, et al., 1995; Robin et al., 1991, 1992). This method is designed to be sensitive to the 
maximum pressure the subject can produce; however, the average rating across three trials (e.g., Robinovitch et al., 1991) may better reflect typical performance. Measures of tongue strength-defined as the highest IOPI measure $\left(P_{\max }\right)$ as well as the average IOPI measure $\left(P_{\text {ave }}\right)$-were included in the present study to examine whether one measure was more useful than the other for predicting oral phase swallowing function.

Swallowing Function. Oral phase swallowing function was evaluated during a clinical ("bedside") or videofluorographic assessment while the participant swallowed standard materials (e.g., of liquid, puree, and/or solid consistency). Whereas the relative reliability of judgments made during clinical versus videofluorographic assessments remains controversial (Martin-Harris, Logemann, McMahon, Schleicher, \& Sandidge, 2000; Splaingard, Hutchens, Sulton \& Chaudhuri, 1988), the method of assessment used in our study was determined based on the standard clinical practices of the cooperating facility. Most inpatient participants underwent clinical assessment, unless the referring physician had requested a videofluorographic procedure. A total of 23 inpatients received

clinical swallowing assessments; the remaining 5 inpatients, and all of the 35 outpatients, were evaluated by using a videofluorograph.

Regardless of the method of assessment used, the swallowing assessment included observation of one or more swallows of materials of several consistencies. Each assessment was conducted according to the unique presentation of the patient. The only experimental control was that the assessment had to allow for the observation of anterior spillage, bolus hold, bolus manipulation, mastication (for patients able to manage solids), and oral clearance. The latter four parameters were chosen because they are thought to be affected by lingual strength and control (Logemann, 1998). Anterior spillage was included as a control variable because it is an oral phase impairment that should not be related to tongue strength, although it is acknowledged that lip and tongue weakness may co-occur. Table 2 describes how each of these characteristics was judged.

For all participants, subjective measures of tongue strength were obtained before the objective measures, to avoid rater bias. In all but five cases, both measures of tongue strength were obtained before the swallowing assessment. For these participants, time constraints related to use of the radiographic suite or other scheduled patient procedures necessitated that the tongue strength measures be obtained after the swallowing assessment.

Ratings of tongue strength and swallowing function were conducted by a single examiner for each participant. Second ratings by the same or a different examiner were conducted at a later time to assess rater reliability. 
TABLE 2. Ratings of oral phase swallowing function.

\begin{tabular}{|c|c|c|c|}
\hline \multirow{2}{*}{$\begin{array}{l}\text { Swallowing } \\
\text { Parameter }\end{array}$} & \multicolumn{3}{|c|}{ Rating Scale } \\
\hline & Left End & Midscale & Right End \\
\hline Anterior spillage & Absent & $\begin{array}{l}\text { Inconsistent } \\
\text { (occurs on less } \\
\text { than half of trials) }\end{array}$ & Consistent \\
\hline Bolus hold & Normal & $\begin{array}{l}\text { Bolus spread } \\
\text { in oral cavity }\end{array}$ & $\begin{array}{l}\text { Premature spillage } \\
\text { into pharynx }\end{array}$ \\
\hline $\begin{array}{l}\text { Bolus manipulation } \\
\text { (liquid, puree, } \\
\text { and solid) }\end{array}$ & Normal & $\begin{array}{l}\text { Impaired ability to move } \\
\text { bolus laterally or } \\
\text { posteriorly }\end{array}$ & $\begin{array}{l}\text { Unable to move } \\
\text { bolus posteriorly }\end{array}$ \\
\hline $\begin{array}{l}\text { Mastication } \\
\text { (solid only) }\end{array}$ & Adequate & Prolonged but adequate & Inadequate \\
\hline Oral clearance & Absent & $\begin{array}{l}\text { Minimal residue (less } \\
\text { than } 1 / 3 \text { of any bolus) }\end{array}$ & $\begin{array}{l}\text { Significant } \\
\text { residue }\end{array}$ \\
\hline
\end{tabular}

\section{Data Analysis}

We documented up to 11 variables for each participant, with 1 variable (objective tongue strength) being measured three times (Table 3 ). The descriptive variable of days postonset was unavailable for 5 participants. Additionally, mastication was not observed for those participants assessed with liquids and purees only. Because most of the planned analyses were univariate or bivariate, they were performed using data for as many of the participants as possible, rather than reducing the sample size to 38 by eliminating all participants for whom some data were missing.

Correlational analyses were conducted using Spearman rank correlations, except between age and IOPI scores, which both provide interval/ratio data. Chi square was used to evaluate relationships between the presence and absence of impairment for two variables in a $2 \times 2$ bivariate frequency table. Finally, $t$ tests and one-way analyses of variance were conducted to examine the differences in age and objective tongue strength in predetermined groups of interest. All significance tests were conducted with an alpha level of .01. 
TABLE 3. List of variables.

\begin{tabular}{ll}
\hline Independent Variables & Dependent Variables \\
\hline Age & Subjective measure of \\
tongue strength & Objective measure of \\
tongue strength & \\
IOPI.1 (first trial) & IOPI.2 (second trial) \\
IOPI.3 (third trial) \\
$P_{\text {max }}$ (maximum of three trials) \\
$P_{\text {avo }}$ (average of three trials) \\
Swallowing measures \\
Presence of oral impairment \\
(of any type) \\
Anterior spillage \\
Bolus hold \\
Bolus manipulation \\
Mastication \\
Oral clearance \\
\hline
\end{tabular}

\section{Reliability}

The unique combination of experienced and inexperienced raters, inpatient and outpatient participants, and clinical and videofluorographic swallowing assessments necessitated complex procedures for assessing the reliability of measures (Table 4). Because most of the inpatient participants were in the acute stages of illness, and thus changes in performance were expected across short intervals of time, it was determined that reliability measures on these participants would be obtained within 36 hours of the first measure. However, the inexperienced raters were not present at the facility on consecutive days. Interrater measures on inpatients were obtained by both experienced and inexperienced examiners, whereas intrarater reliability measures on inpatients were obtained by experienced examiners only. 
TABLE 4. Reliability analysis methods.

\begin{tabular}{lll}
\hline \multirow{2}{*}{ Parameter } & \multicolumn{1}{c}{ Analysis Method } \\
\cline { 2 - 3 } $\begin{array}{l}\text { Subjective and objective } \\
\text { measures of tongue strength }\end{array}$ & \multicolumn{1}{c}{ Intrarater } & \multicolumn{1}{c}{ Interrater } \\
$\begin{array}{l}\text { Swallowing measures obtained during } \\
\text { clinical (bedside) assessments }\end{array}$ & $\begin{array}{l}\text { Measures collected from } \\
\text { inpatients by experienced } \\
\text { examiners }\end{array}$ & $\begin{array}{l}\text { Measures collected from } \\
\text { inpatients only }\end{array}$ \\
$\begin{array}{l}\text { Swallowing measures obtained } \\
\text { during videofluorographic assessments }\end{array}$ & $\begin{array}{l}\text { Mellected from } \\
\text { examiners }\end{array}$ & \multicolumn{1}{c}{$\begin{array}{c}\text { Measures collected from } \\
\text { inpatients only }\end{array}$} \\
\hline
\end{tabular}

Outpatients were available for assessment only on the day they were evaluated. This had several implications for the reliability measures. First, we were unable to collect second measures of tongue strength from this group, so reliability measures for tongue strength were conducted using inpatient data only. However, the outpatient swallowing measures were included in the reliability calculations because the video recordings could be reviewed at a later time.

For subjective measures of tongue strength and measures of oral phase swallowing function, trial-to-trial agreement was examined. For objective measures of tongue strength, the highest pressures $\left(P_{\max }\right)$ obtained during the first and second evaluations were correlated.

Subjective Judgments of Tongue Strength. Reliability results are reported in Table 5 . Of the 35 participants whose tongue strength was rated by experienced examiners, 9 were selected for reevaluation by the same examiner to assess intrarater reliability. Of these, 8 obtained the same rating as they had initially. For the remaining participant, the first and second ratings were within one rating interval of each other. The ratings of 7 participants were selected for reevaluation by a second examiner to assess interrater reliability. All of these ratings were in agreement. 
TABLE 5. Reliability results.

\begin{tabular}{lcc}
\hline & \multicolumn{2}{c}{ Reliability Measures } \\
\cline { 2 - 3 } \multicolumn{1}{c}{ Parameter } & Intrarater & Interrater \\
\hline Subjective measure & $89 \%$ & $100 \%$ \\
of tongue strength & & \\
IOPI $\left(P_{\max }\right)$ & $r=.97$ & $r=.95$ \\
Anterior spillage & $100 \%$ & $94 \%$ \\
Bolus hold & $75 \%$ & $94 \%$ \\
Bolus manipulation & $93 \%$ & $100 \%$ \\
$\quad$ (liquid, puree, and solid) & & \\
Mastication (solid only) & $100 \%$ & $100 \%$ \\
Oral clearance & $100 \%$ & $94 \%$ \\
\hline
\end{tabular}

Objective Measures of Tongue Strength. The reliability of the objective instrument was assessed by computing correlations between the highest IOPI measurements $\left(P_{\max }\right)$ obtained during the first and second evaluations. When the measures were obtained by the same examiner (12 participants), the correlation between first and second $P_{\max }$ was .97. The same correlation for measures obtained by two different examiners (5 participants) was .95 .

Measures of Oral Phase Swallowing Function. Five parameters of oral phase swallowing function were targeted: anterior spillage, bolus hold, bolus manipulation, mastication, and oral clearance. For each parameter, item-to-item agreement was calculated. Table 5 shows that interrater and intrarater reliability was greatest for the mastication parameter and poorest for bolus hold. In all cases, however, there was nearly $100 \%$ agreement regarding the existence of difficulty. The lower reported agreement levels resulted from disagreement regarding the severity of the impairment.

\section{Results}

\section{Subjective Measures of Tongue Strength}

Figure 2 illustrates the number of participants who received the various ratings of tongue strength. Fifty percent of the participants were judged to have normal tongue strength. The remaining participants were evenly distributed across the weakness rating levels. No differences in subjective ratings of tongue strength were observed with respect to gender ( $t=.17, p=.87, d=.041)$. Tongue strength was related to age only for those participants judged to exhibit tongue weakness, $F(2,23)=$ $6.73, p=.005, f=.51$ (Figure 3 ). Follow-up mean comparisons revealed that participants rated as severely weak were significantly older than those rated as slightly weak. Confidence bands for ratings other than normal or severely 
weak overlapped, hence the lack of significant differences between these groups.

FIGURE 2. Subjective ratings of tongue strength for men and women.

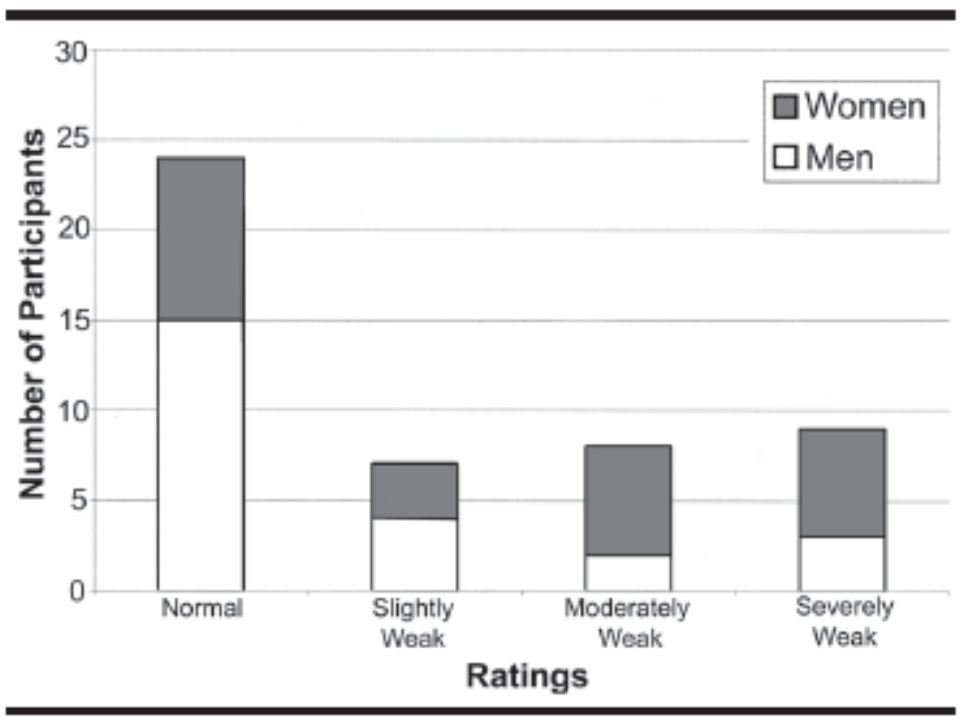

FIGURE 3. Mean age of participants for each subjective rating of tongue strength.

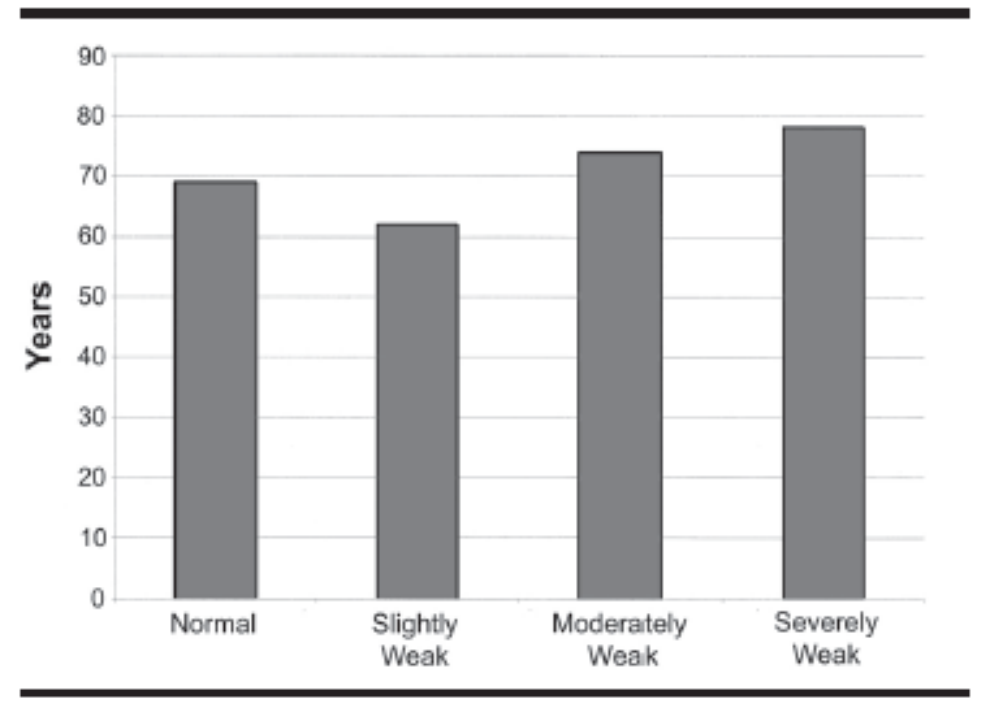

\section{Objective Measures of Tongue Strength}

The mean tongue strength measures calculated using $P_{\max }$ and $P$ ave pressures are included in Table 6. The correlation between these two measures was quite high, $r=.976$. No statistically significant differences in objective measures of tongue strength by gender were seen for 
either $P_{\max }(t=.62, p=.54, d=.160)$ or $P_{\text {ave }}(t=.55, p=$ $.59, d=.142$ ) (Figure 4). A weak but statistically significant negative relationship was observed between $P_{\max }$ and age $(r=-.463, p=.000)$ and between $P$ ave and age $(r=$ $-.407, p=.001)$, indicating that older participants tended to produce lower tongue pressures.

FIGURE 4. Objective measures of tongue strength for men and women.

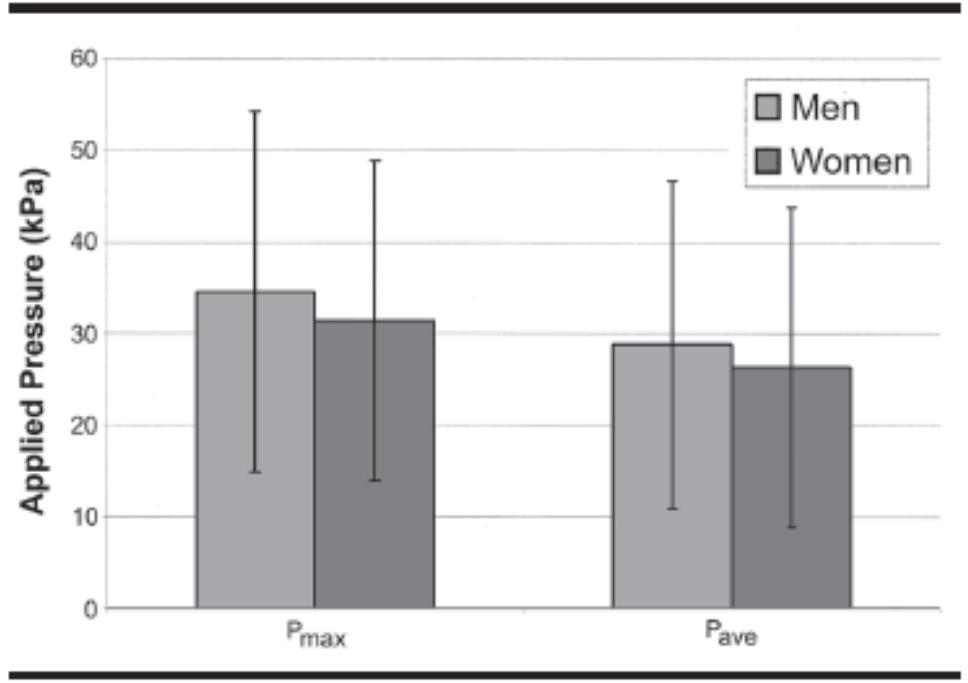

FIGURE 5. Mean IOPI scores by subjective ratings of tongue strength.

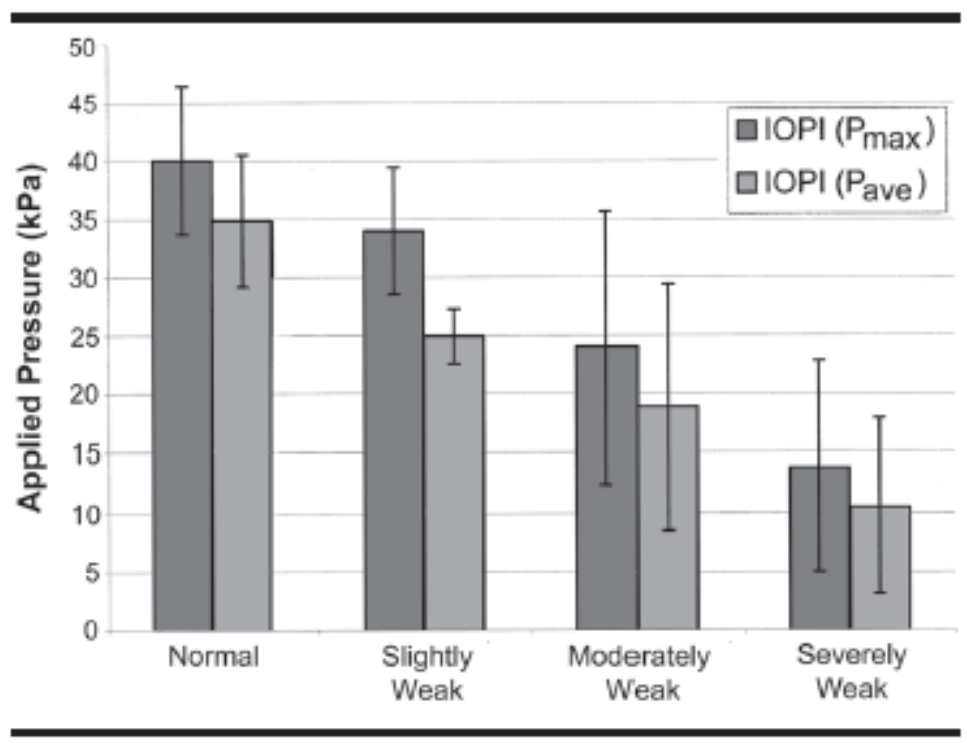




\section{Relationship Between Subjective and Objective Measures of Tongue Strength}

The correlation between subjective ratings and $P_{\max }$ score was $.541(p=.000)$, indicating that $P_{\max }$ Scores tended to decrease as tongue strength was rated as weaker. A similar relationship was observed for subjective ratings and $P$ ave $(r=.567, p=.000)$. Figure 5 illustrates the mean $P_{\max }$ and $P_{\text {ave }}$ for each of the subjective ratings. One-way analysis of variance revealed a significant effect of subjective rating for both $P_{\max }, F(3,52)=7.25, p=.000, f$ $=.388$ and $P_{\text {ave, }} F(3,52)=8.26, p=.000, f=.44$. The confidence bands shown in Figure 5 show that IOPI measures could only differentiate the group rated as severely weak from the group rated as normal. Although the differences in the mean ratings between these two groups were both statistically and clinically significant, the observed moderate effect size reflects the overlap in IOPI scores evident across the other groups.

We further examined whether the relationship between subjective and objective measures of tongue strength was affected by the experience of the examiner. For the inexperienced raters, the correlation between subjective rating and $P_{\max }$ was .696 $(p=.001)$, and between subjective rating and $P$ ave was .719 $(p=.000)$. In contrast, the same correlations for experienced raters were $.395(p=$ $.017)$ and $.413(p=.011)$, respectively.

TABLE 6. Tongue strength as measured objectively with IOPI.

\begin{tabular}{lcccc}
\hline $\begin{array}{c}\text { IOPI } \\
\text { Measure }\end{array}$ & Minimum & Maximum & $M$ & $S D$ \\
\hline IOPI.1 & 1 & 69 & 26.70 & 16.78 \\
IOPI.2 & 1 & 78 & 28.37 & 18.61 \\
IOPI.3 & 1 & 75 & 26.97 & 17.58 \\
$\mathrm{P}_{\max }$ & 2 & 78 & 32.75 & 18.44 \\
$\mathrm{P}_{\text {ava }}$ & 1 & 64 & 27.30 & 16.67 \\
\hline IOPI.1 = first trial; IOPI.2 = second trial; IOPI.3 = third trial; $P_{\max }=$ \\
highest trial; $P_{\text {avo }}=$ average of three trials. \\
\hline
\end{tabular}

\section{Oral Phase Swallowing Impairments}

The numbers of participants identified with impairments of each of the parameters of oral phase function are listed in Table 7. The most common impairments were in the areas of bolus hold and manipulation. Anterior spillage, impaired mastication, and oral residue were observed the least frequently. A total of $32(51 \%)$ of all participants were identified as being impaired in at least one of the 
parameters. For the remainder of this discussion, these participants will be designated as "those with oral phase swallowing impairments."

The mean age of participants with oral phase swallowing impairments was 66.6 years $(S D=11.2)$, whereas the mean age of participants without oral phase swallowing impairments was 73.8 years $(S D=16)$. This age difference was not significant $(t=2.07, p=.044, d=.51)$, although the effect size was moderate.

TABLE 7. Frequency of oral impairments.

\begin{tabular}{lc}
\hline Parameter & $\begin{array}{c}\text { Number of Participants } \\
\text { Exhibiting Impairment }\end{array}$ \\
\hline Anterior spillage & 13 \\
Bolus hold & 21 \\
Bolus manipulation & 20 \\
$\quad$ (liquid, puree, and solid) & 13 \\
Mastication (solid only) & 13 \\
Oral clearance & \\
$\quad$ Total number of participants & 32 \\
exhibiting any oral phase impairment & \\
\hline
\end{tabular}

\section{Relationship Between Measures of Tongue Strength and Oral Phase Swallowing Function}

Because the relationships between subjective and objective measures of tongue strength were different for experienced and inexperienced examiners, we looked at the relationships between subjective ratings and swallowing function separately for each of these groups. Table 8 lists the rank correlations between each tongue strength measure and each oral phase swallowing measure for experienced and inexperienced raters. Subjective ratings of tongue strength made by experienced raters were most closely related to bolus manipulation and mastication, and least related to anterior spillage. For inexperienced raters, bolus hold and bolus manipulation were most highly correlated with subjective measures of tongue strength. The pattern of relationships between strength and oral phase measures was slightly different when tongue strength was measured objectively. $P_{\max }$ was weakly correlated with bolus manipulation but was not strongly related to any of the other variables. Pave was related to bolus manipulation and, to a lesser extent, mastication. In all cases, the subjective measures were more highly correlated with the oral phase swallowing measures than were the objective measures. 
We further examined the relationship between subjective rating of tongue strength and oral phase swallowing function by computing the rank correlation between subjective rating and the presence or absence of any oral phase swallowing impairment. For experienced raters, this correlation was $-.732(p=.000)$, whereas for inexperienced raters the correlation was $-.625(p=.000)$. When the data are further collapsed so that participants are categorized by the presence or absence of oral phase impairment and by the presence or absence of reduced tongue strength (Figure 6), the chi square analysis indicates a strong relationship between the presence of tongue weakness and the presence of oral phase swallowing impairment $\left(\mathrm{X}_{2}=\right.$ 33.603, $p=.000, \omega=.73$ ).

Similarly, we examined the mean IOPI scores for participants with and without oral phase swallowing impairments (Figure 7). Individuals with oral phase swallowing impairments exhibited significantly lower mean $P_{\max }$ and $P_{\text {ave }}$ than participants without oral phase impairments $\left(t=4.11, p=.0001, d=.98\right.$ for $P_{\max } ; t=4.44$, $p=.0001, d=1.02$ for $\left.P_{\text {ave }}\right)$.

FIGURE 6. Subjective measures of tongue strength and oral phase swallowing impairments.

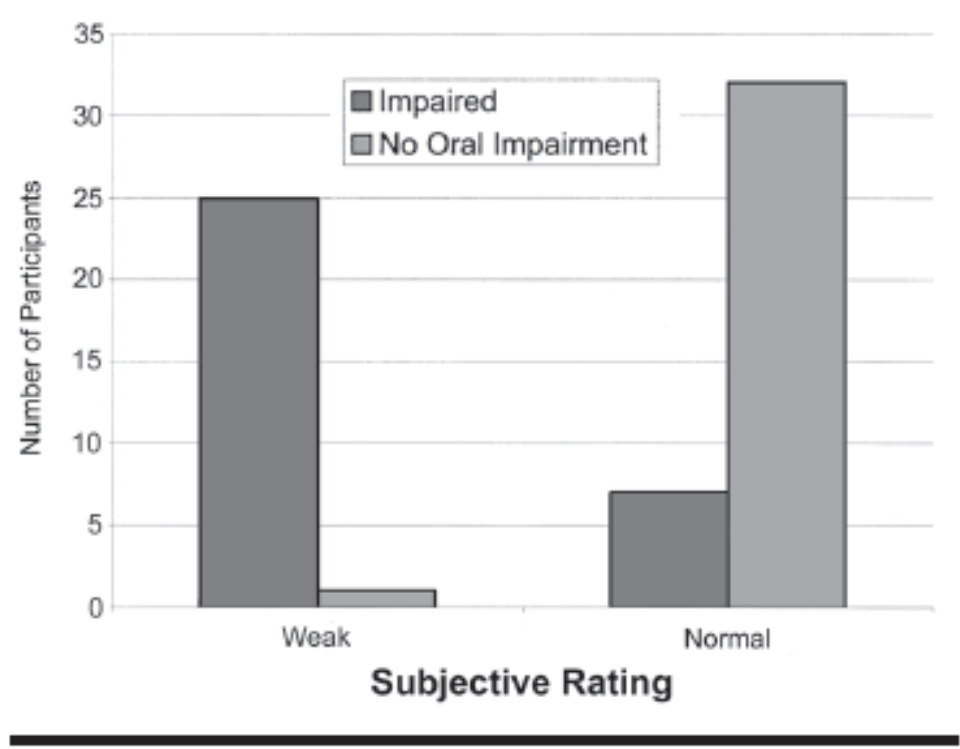


FIGURE 7. Objective measures of tongue strength and oral phase swallowing impairments.

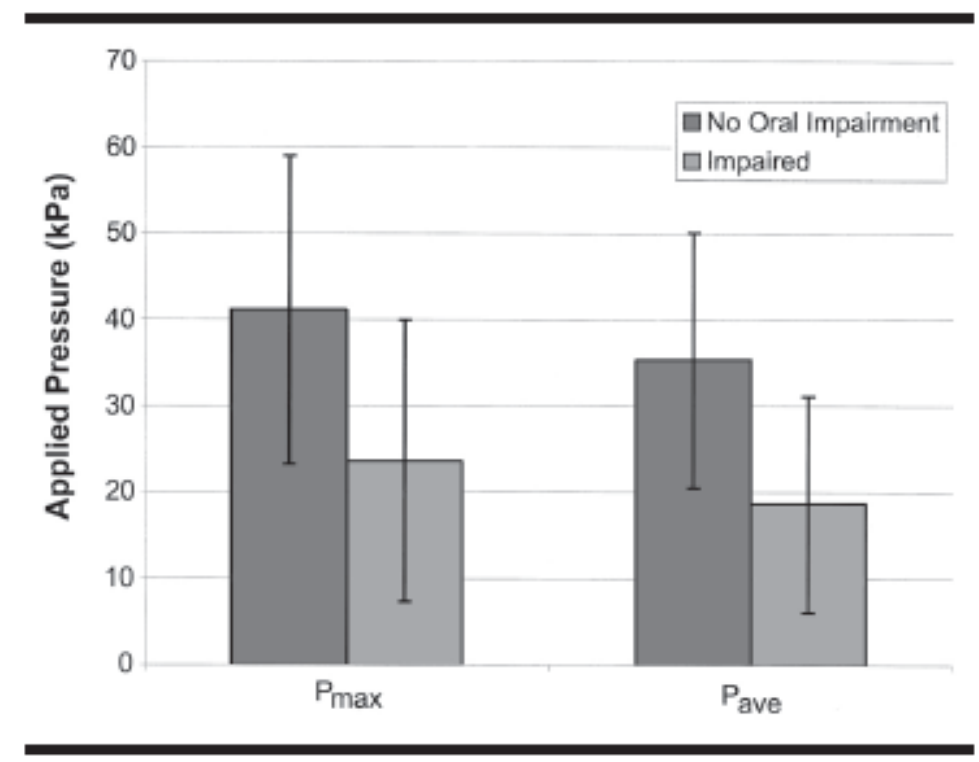

\section{Discussion}

This study was conducted to investigate two issues: (a) the relationships among subjective and objective measures of tongue strength and various aspects of oral phase swallowing function, and (b) how these relationships are affected by examiner experience.

\section{Relationship Between Subjective and Objective Measures of Tongue Strength}

Subjective and objective measures of tongue strength were weakly correlated, with significant differences in $P_{\max }$ noted between participants whose tongue strength was rated as normal and those rated as severely weak. An obvious explanation for the discrepancy between the objective and subjective measures is that the movements involved in the two strength measures differed. The IOPI measure involved a single lingual movement (anterior elevation), whereas the subjective rating assessed force during protrusion and lateralization. The weak relationships observed may reflect the co-occurrence of weakness in several lingual muscle groups.

Perhaps more interesting is the difference in correlations between objective and subjective measures for experienced and inexperienced examiners. Although not stated explicitly a priori, we had anticipated that the experienced and 
inexperienced raters' judgments of tongue strength would be similarly related to the objective measures, or that the experienced raters' judgments would be more highly predictive of the objective measures. The finding that experienced raters' subjective judgments were so weakly related to the objective measures was unexpected, and obvious explanations are lacking.

One possibility is that the inexperienced and experienced raters attended to different components of the movement tasks when developing their subjective ratings. Specifically, the experienced raters may have applied more significance to lateral movements than did the inexperienced raters. This explanation is appealing for two reasons. First, tongue protrusion shares several movement features with tongue elevation, including bilateral contractions of the lingual musculature. Ratings based heavily on protrusion would be expected to correlate more highly with the IOPI measure. Second, lateral tongue movements are arguably more important to the oral phase functions of bolus manipulation and mastication. That the experienced raters' judgments correlated most strongly with these variables suggests that their judgments may have been heavily influenced by forces produced during lateral movements.

For both experienced and inexperienced raters, the observed correlations between subjective and objective measures were low enough to support the assertion that both objective and subjective measures of tongue strength offer a unique contribution to impairment-level assessment of swallowing function. Subjective measures have an apparent advantage over current clinically available objective measures that do not allow the examiner to assess tongue strength during lateralization. Thus, subjective measures may better predict specific aspects of swallowing function. However, objective measures may be more sensitive to small differences in strength observed during recovery or disease progression (Luschei, 1991; Reddy et al., 1990; Robinovitch et al., 1991; Sukthankar et al., 1994).

Additional research is needed to determine precisely how the subjective judgments of experienced raters differ from those of inexperienced raters. Specifically, studies incorporating separate subjective ratings of protrusion, lateralization, and/or anterior elevation may reveal differences among raters. Additionally, it would be interesting to know if explicit training in rating tongue strengthperhaps using reference forces (e.g., "This is severely weak; this is very strong.")-would improve the agreement between experienced and inexperienced raters. [2] 
TABLE 8. Relationship between oral phase impairments and tongue strength measures.

\begin{tabular}{|c|c|c|c|c|}
\hline \multirow[b]{2}{*}{$\begin{array}{c}\text { Oral Phase } \\
\text { Swallowing Parameter }\end{array}$} & \multicolumn{2}{|c|}{ Subjective Rating } & \multirow[b]{2}{*}{$P_{\max }$} & \multirow[b]{2}{*}{$P_{\text {awo }}$} \\
\hline & $\begin{array}{l}\text { Experienced } \\
\text { Rater }\end{array}$ & $\begin{array}{l}\text { Inexperienced } \\
\text { Rater }\end{array}$ & & \\
\hline Anterior spillage & $-.487^{*}$ & -.185 & $-.317^{*}$ & $-.318^{*}$ \\
\hline Bolus hold & $-.514^{*}$ & $-.528^{*}$ & $-.356^{*}$ & $-.327^{*}$ \\
\hline Bolus manipulation & $-.787^{* *}$ & $-.584^{* *}$ & $-.558^{* *}$ & $-.555^{* *}$ \\
\hline Mastication & $-.792^{* *}$ & $-.381^{*}$ & $-.367^{*}$ & $-.427^{*}$ \\
\hline Oral clearance & $-.740^{* *}$ & $-.415^{*}$ & -.244 & -.262 \\
\hline
\end{tabular}

${ }^{*} p<.01,{ }^{*} p<.001$

\section{Relationships Between Subjective Ratings of Tongue Strength and Oral Phase Swallowing Function}

Because experienced and inexperienced raters judged tongue strength differently, it was necessary to examine the relationship between subjective measures of tongue strength and oral phase swallowing function separately for these two groups. As can be seen in Table 8, experienced raters' judgments of tongue strength were more highly correlated with the various measures of oral phase swallowing function (with the exception of bolus hold) than were the inexperienced raters' judgments. The experienced raters' judgments were most highly correlated with the oral phase impairments of bolus manipulation and oral clearance, but exhibited statistically significant correlations with all of the oral phase impairments studied. In contrast, the inexperienced raters' judgments were most highly related to measures of bolus hold and bolus manipulation.

We predicted that subjective ratings of tongue strength, because they incorporate strength measured during lateral tongue movements, would be associated with oral phase swallowing measures such as bolus manipulation. Our findings support this prediction, particularly for the experienced examiners. We further predicted that measures of tongue strength would not be highly related to anterior spillage, because this oral function relies more on adequate labial rather than lingual function. Although this prediction held true for the inexperienced raters, the experienced raters' judgments of tongue strength were weakly correlated with anterior spillage. This finding may reflect the co-occurrence of orofacial and lingual deficits for some of the participants.

Examining the ratings of experienced and inexperienced raters together, it was found that 7 of 34 participants with oral phase dysphagia were judged to have normal tongue 
strength. This finding is understandable in that reduced tongue strength is clearly not the only potential cause of oral phase swallowing dysfunction. However, subjective measures of tongue strength appear to be highly specific predictors for oral phase dysphagia. Only one participant identified as exhibiting tongue strength below the normal range demonstrated normal oral phase swallowing function. This suggests that patients identified as having tongue weakness during an oral-motor exam are at high risk for oral phase swallowing disorders.

\section{Relationships Between Objective Measures and Oral Phase Swallowing Function}

Objective measures of tongue strength were not as highly correlated with oral phase swallowing function as were the subjective measures. This is not surprising because the objective measure targeted a single lingual movement. The subjective measure, which incorporated several lingual movements, was more likely to incorporate the movements associated with multiple swallowing components. We predicted that the objective measure, which measured tongue strength during an anterior/ superior movement, would be more highly related to measures of oral clearance resulting from inadequate bolus propulsion. In fact, oral clearance was the variable most weakly correlated with objective measures of tongue strength, even though it was strongly related to subjective ratings of tongue strength made by experienced examiners. It is likely that the operational definition of oral clearance was not specific enough in the present study to be sensitive to oral propulsion impairments only. That is, oral residue may result when parts of the bolus are lost in the oral cavity as a result of poor bolus hold or manipulation. Because we did not differentiate between residue related to poor control and that resulting from poor propulsion, potentially significant relationships might have been overlooked. Future investigations should define this variable with greater sensitivity to the differences in types and/or location of oral residue. Further, studies controlling for the method of swallowing assessment (e.g., clinical versus videofluorographic) may reveal more details regarding the relationship between tongue strength and oral phase swallowing function.

Although the objective measure of tongue strength was not observed to be a strong predictor of specific oral phase impairments, it did predict well the presence of any oral phase impairment in the groups of participants. We examined the objective tongue strength data more closely to determine if a "cutoff score" or a minimum IOPI score necessary for adequate oral phase function could be identified. Figure 8 illustrates the considerable overlap that exists between the participants with and without oral phase 
impairments. However, Robbins et al. (1995) reported that both young and normally aging adults generated tongue pressures of approximately $20 \mathrm{kPa}$ at the tongue tip during saliva swallows. On the basis of their report, we might expect participants who were unable to produce maximum tongue pressures of $20 \mathrm{kPa}$ to exhibit oral phase dysphagia. In the present study, 13/17 (76\%) of participants whose maximum tongue strength measured $20 \mathrm{kPa}$ or below exhibited oral phase dysphagia. Similarly, $5 / 32(16 \%)$ of participants without oral phase impairments exhibited tongue strength measures $20 \mathrm{kPa}$ or below. This suggests that an IOPI score cutoff of $20 \mathrm{kPa}$ would be more specific than it is sensitive to the presence of oral phase swallowing impairments in individual participants.

Our data suggest that both subjective and objective measures of tongue strength are predictive of the presence of oral phase dysphagia. However, additional study is needed to determine precisely how lingual weakness affects swallowing function. The relationships observed may reflect the impact of generally reduced function on both tongue strength and oral phase swallowing function. Brainstem dysfunction, as well as systemic impairments such as pneumonia or degenerative disease, may contribute to deficits in both strength and function. Additional support for the unique contribution of lingual weakness to swallowing dysfunction would be provided by evidence of improved function following strength training (e.g., Solomon \& Stierwalt, 1995) during appropriately controlled treatment studies.

FIGURE 8. Distribution of $P_{\max }$ for participants with and without oral phase swallowing impairments.

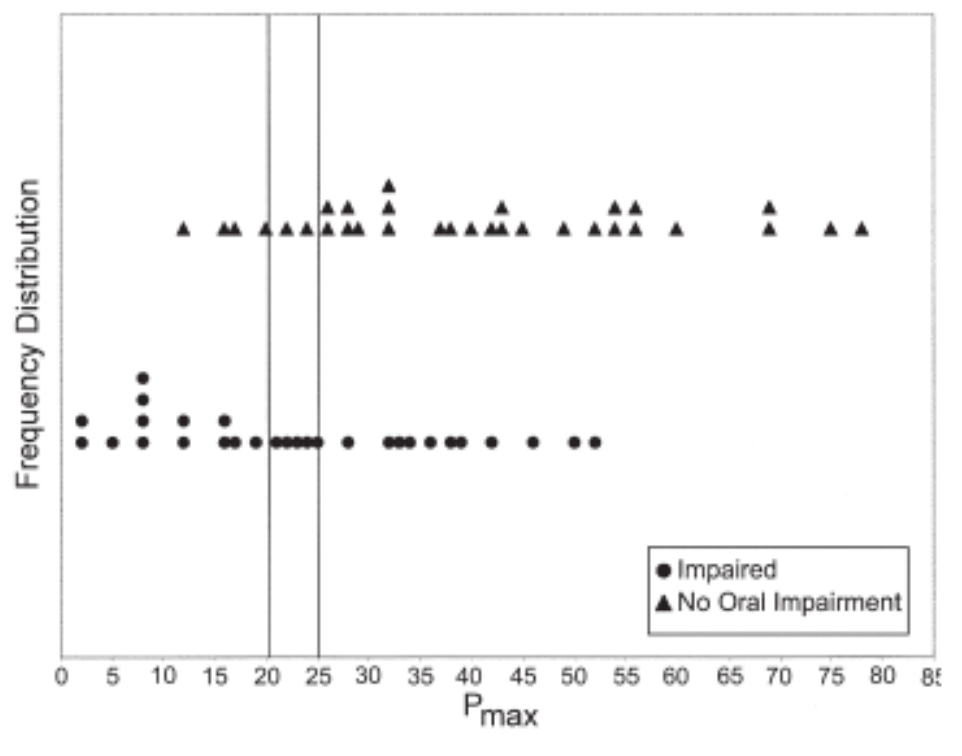




\section{Additional Discussion}

Selection of Subjective Measures of Tongue Strength. Examiners in this study subjectively rated tongue strength during lingual protrusion and lateralization according to the clinical protocol of the cooperating facility. However, it is likely that different relationships between subjective and objective measures of tongue strength, as well as between tongue strength and oral phase swallowing function, would have been observed if strength had been rated during different lingual movements. For example, subjective ratings made during anterior elevation may be more highly correlated with IOPI measures than were the ratings recorded during the present study. Further, subjective ratings of individual lingual movements (rather than those incorporating several movements) will likely reveal a different pattern of relationships than reported here. Nonetheless, the current findings add to the growing literature suggesting that lingual strength measured during lateral tongue movements may be particularly useful in assessing the level of impairment of swallowing function (Reddy et al., 1990; Robinovitch et al., 1991).

Selection of Objective Measures of Tongue Strength. If the strength of lateral tongue movements is indeed relevant to the assessment of swallowing function, current clinically available tools for the objective measurement of tongue strength that measure strength only during lingual elevation are ill-suited for this purpose. As additional evidence accumulates to support the use of impairment-level observations in the diagnosis and management of swallowing dysfunction, clinical instrumentation will need to evolve in line with these findings.

With respect to whether $P_{\max }$ or $P_{\text {ave }}$ provides the better operational definition of tongue strength, our study indicates that both measures relate similarly to subjective measures of tongue strength and oral phase swallowing function. Thus, it does not appear that one definition is more clinically useful than the other. From a practical standpoint, using $P_{\max }$ may be more efficient in a clinical setting because no calculation is required. Future research that incorporates both of these values (or alternative operational definitions, such as a measure of variability) may reveal particular clinical uses for which one measure is preferred over the other.

It is tempting, but nonetheless inappropriate, to extrapolate from studies such as these-which demonstrate a relationship between weakness and impaired performancethat strength training will improve performance.

Additional treatment research is needed in this area. However, a beginning literature supports the use of strength training of the tongue to improve oral phase swallowing function (Solomon \& Stierwalt, 1995; Stierwalt \& Robin, 1996). Studies are needed to examine 
the parameters of strength and/or endurance training that result in the greatest improvements in oral phase swallowing function and to identify the individuals for whom strength training is most appropriate.

In summary, our study found that subjective ratings of tongue strength made by experienced examiners are differentially correlated to bolus manipulation, mastication, and oral clearance. The subjective ratings of inexperienced examiners, as well as objective measures of tongue strength, are good predictors of the presence of oral phase dysphagia, but they are not as sensitive to specific aspects of swallowing function. These findings suggest the need for continued research regarding the relationships among impairment and activity level measures of function. Future studies should address the usefulness of both subjective and objective measures of impairment, as well as a variety of functional measures of speech and swallowing ability.

\section{Acknowledgments}

This work was supported by a grant from the Appalachian State University Research Council. We gratefully acknowledge the contributions of the graduate students who participated in the project, and of the patients who allowed us to include their assessment results in our study.

\section{References}

Crow, H. C., \& Ship, J. A. (1996). Tongue strength and endurance in different aged individuals. Journal of Gerontology, 51, 247-250.

Duffy, J. R. (1995). Motor speech disorders: Substrates, differential diagnosis, and management. St. Louis, MO:

Mosby.

Logemann, J. A. (1998). Evaluation and treatment of swallowing disorders (2nd ed). Austin, TX: Pro-Ed.

Luschei, E. S. (1991). Development of objective standards of nonspeech oral strength and performance. In C. Moore, $\mathrm{K}$. Yorkston, \& D. Beukelman (Eds.), Dysarthria and apraxia of speech: Perspectives on management (pp. 3-14). Baltimore: Brookes.

Martin-Harris, B. S., Logemann, J. A., McMahon, S., Schleicher, M., \& Sandidge, J. (2000). Clinical utility of the modified barium swallow. Dysphagia, 15, 11-20.

Reddy, N. P., Costarella, B. R., Grotz, R. C., \& Canilang, E. P. (1990). Biomechanical measurements to characterize the oral phase of dysphagia. IEEE Transactions on Biomedical Engineering, 37, 392-397.

Robbins, J., Levine, R., Wood, J., Roecker, E. B., \& Luschei, E. (1995). Age effects on lingual pressure generation as a risk factor for dysphagia. Journal of Gerontology, 50A, M257M262.

Robin, D. A., Goel, A., Somodi, L. B., \& Luschei, E. S. (1992). Tongue strength and endurance: Relation to highly skilled movements. Journal of Speech and Hearing Research, 35, 1239-1245. 
Robin, D. A., \& Luschei, E. S. (1992). Reference manual for the lowa Oral Performance Instrument. Oakdale, IA: Breakthrough.

Robin, D. A., Somodi, L. B., \& Luschei, E. S. (1991). Measurement of tongue strength and endurance in normal and articulation disordered subjects. In C. Moore, K. Yorkston, \& D.

Beukelman (Eds.), Dysarthria and apraxia of speech: Perspectives on management (pp. 173-184). Baltimore: Brookes.

Robinovitch, S. N., Herschler, D., \& Romilly, D. P. (1991). A tongue force measurement system for the assessment of oralphase swallowing disorders. Archives of Physical Medicine and Rehabilitation, 72, 38-42.

Solomon, N. P., Lorell, D. M., Robin, D. A., Rodnitzky, R. L., \& Luschei, E. S. (1995). Tongue strength and endurance in mild to moderate Parkinson's disease. Journal of Medical Speech Language Pathology, 3, 15-26.

Solomon, N. P., Robin, D. A., \& Luschei, E. S. (2000). Strength, endurance, and stability of the tongue and hand in Parkinson Disease. Journal of Speech, Language, and Hearing Research, 43, 256-267.

Solomon, N. P., \& Stierwalt, J. A. G. (1995, December). Strength and endurance training for dysarthria. ASHA Special Interest Division 2 Newsletter, 5(2), 13-16.

Splaingard, M. B., Hutchens, B., Sulton, L., \& Chaudhuri, G. (1988). Aspiration in rehabilitation patients: Videofluoroscopy vs. bedside clinical assessment. Archives of Physical Medicine and Rehabilitation, 69, 637-640.

Stierwalt, J. A. G., \& Robin, D. A. (1996). Tongue strengthening in the treatment of severe flaccid dysarthria: A singlesubject study. Poster presented at the 1996 Motor Speech Conference, Amelia Island, FL.

Stierwalt, J. A. G., Robin, D. A., Solomon, N. P., Weiss, A. L., \& Max, J. E. (1996). Tongue strength and endurance: Relation to speaking ability of children and adolescents following traumatic brain injury. In D. A. Robin, K. M. Yorkston, \& D. R. Beukelman (Eds.), Disorders of motor speech: Assessment, treatment, and clinical characterization.

Baltimore: Brookes.

Sukthankar, S. M., Reddy, N. P., Canilang, E. P., Stephenson, L., \& Thomas, R. (1994). Design and development of portable biofeedback systems for use in oral dysphagia rehabilitation. Medical Engineering and Physics, 16, 430-435. 